\title{
Performance of Ultrasensitive Rapid Diagnostic Tests for Detecting Asymptomatic Plasmodium falciparum
}

\author{
Shunmay Yeung, ${ }^{1 *}$ David McGregor, ${ }^{1}$ Nicola James, ${ }^{1}$ Soy Ty Kheang, ${ }^{2}$ Saorin Kim, ${ }^{3}$ Nimol Khim, ${ }^{3}$ Po Ly, ${ }^{4}$ \\ Siv Sovannaroth, ${ }^{4}$ and Benoit Witkowski ${ }^{3}$ \\ ${ }^{1}$ Clinical Research Department, Faculty of Infectious and Tropical Disease, Malaria Centre, London School of Hygiene and Tropical Medicine, \\ London, United Kingdom; ${ }^{2}$ Health and Social Development (HSD), Phnom Penh, Cambodia; ${ }^{3}$ nstitut Pasteur du Cambodge, Phnom Penh, \\ Cambodia; ${ }^{4}$ National Center for Parasitology, Entomology and Malaria Control, Phnom Penh, Cambodia
}

\begin{abstract}
Proposed interventions for eliminating drug-resistant Plasmodium falciparum malaria include the targeting of asymptomatic carriers through screening and treatment. We report on the diagnostic performance of the recently developed ultrasensitive rapid diagnostic test (URDT) compared with screening with conventional RDTs (cRDT) and polymerase chain reaction (PCR) under field conditions in Cambodia in a total of 2,729 individuals. The $P$. falciparum positivity by quantitative PCR (qPCR) was 3.8\% (26/678) in those screened during active case detection and $0.5 \%$ $(10 / 2,051)$ in the cross-sectional survey. Compared with qPCR, the sensitivity of the uRDTs was $53.8 \%(95 \% \mathrm{Cl}$ : 33.4-73.4\%) when used in active case detection and 60.0\% (95\% Cl: 26.2-87.8\%) in the cross-sectional survey. The uRDTs did not show a significant improvement in diagnostic performance over cRDTs when used for active case detection and for a malaria prevalence survey in the context of this low-transmission setting.
\end{abstract}

Like elsewhere in Southeast Asia, the burden of malaria in Cambodia has decreased dramatically over the last 15 years. ${ }^{1,2}$ However, the region is under the threat of Plasmodium falciparum resistance to artemisinin-based combination therapy. ${ }^{3}$ The Royal Government of Cambodia has committed to eliminating $P$. falciparum malaria by 2020 and all forms of malaria by $2025 .^{4}$ Neighboring countries including Thailand, Vietnam, and Laos have also committed to malaria elimination.

To eliminate $P$. falciparum malaria, treatment strategies that solely focused on patients presenting with symptomatic malaria are unlikely to be sufficient. Asymptomatic carriage with low-level parasitaemias has now been well documented in the region and represents an important potential reservoir of infection. ${ }^{5}$

Until recently, efforts to screen for low-density infections have been limited by the available diagnostics. Conventional rapid diagnostic tests (cRDTs), although easy to use in the field and sufficiently sensitive for confirming infection in symptomatic infections, are not sensitive enough to detect low-level parasitaemias in asymptomatic infections. Conversely, molecular diagnosis using polymerase chain reaction (PCR) is highly sensitive but requires sophisticated laboratory facilities and, therefore, cannot be used operationally as a screening tool in remote forested areas where populations at a risk of malaria infection live. ${ }^{6-8}$

New "ultrasensitive rapid diagnostic tests" (uRDTs) have recently been developed and offer the exciting potential of a field-deployable highly sensitive test. Similar to cRDTs, they are based on the immunodetection of histidine-rich protein 2 (HRP2); however, they have a reported sensitivity of more than $50 \%$ with $P$. falciparum densities between 0.1 and $1 \mathrm{p} / \mu \mathrm{L}$ under laboratory conditions. ${ }^{9}$ Although promising, this new test needs to be evaluated under field conditions to determine

\footnotetext{
*Address correspondence to Shunmay Yeung, Clinical Research Department, London School of Hygiene and Tropical Medicine, Keppel St., London WC1E 7HT, United Kingdom. E-mail: shunmay.
} yeung@Ishtm.ac.uk its potential performance and utility in malaria elimination programs.

We aimed to evaluate the performance of URDT in comparison with a cRDT and quantitative PCR (qPCR) under field conditions on the Cambodian-Thai border.

This study was nested within a large study of active case detection for malaria, which was carried out in Oddar Meanchey Province in Northwest Cambodia. In brief, in the 65 villages in the intervention arm, active case detection was carried out both reactively and proactively. Reactive case detection was carried out around "index cases," that is, patients with symptomatic $P$. falciparum malaria who had presented to a village malaria worker or health center. Screening for malaria was carried out on members of their household, individuals who had traveled to the forest with them ("co-travelers"), and "high-risk" neighbors. High-risk individuals were defined as anyone reporting to have slept overnight in the forest in the past month and any individual with a reported fever in the previous 48 hours. In proactive case detection, all high-risk individuals in villages with high number of $P$. falciparum cases were screened proactively.

From June to December 2017, screening for malaria was carried out using three diagnostic tests: URDT (Alere Malaria Ag P.f, Abbott, Lake Bluff, IL), cRDT under routine use (Standard Diagnostics, Bioline, Gyeonggi-do, Republic of Korea) with HRP-Il and pLDH antigen RDT (Alere Malaria Ag P.f, Abbott), and nested PCR on blood spot samples conducted at the Institute Pasteur laboratory in Phnom Penh, as previously described. ${ }^{10}$ In addition, a cross-sectional survey was conducted from November to December 2017 in 17 of the highest burden villages. All participants found positive for malaria by any diagnostic tests were offered treatment with the first-line artemisinin-based combination therapy.

Both village malaria workers and survey data collectors were provided with training on how to take blood samples and carry out RDTs correctly as well as preparing filter paper and preprepared 96-well plates for subsequent PCR analysis. Nucleic material extraction from dried blood spot and screening for any malaria species infection and then specific $P$. falciparum infection were carried out according to the methodology of Canier et al. ${ }^{11}$ Results of RDTs were recorded 
TABLE 1

Comparison between PCR and cRDTs and uRDTs for screening for Plasmodium falciparum during active case detection and the crosssectional survey

\begin{tabular}{|c|c|c|c|c|}
\hline & cRDT+ & CRDT- & uRDT+ & URDT- \\
\hline \multicolumn{5}{|c|}{ Active case detection } \\
\hline Total & 24 & 654 & 35 & 643 \\
\hline PCR Pf+ & 12 & 14 & 14 & 12 \\
\hline PCR Pf- & 12 & 640 & 21 & 631 \\
\hline Sensitivity (\%) & \multicolumn{2}{|c|}{$\begin{array}{c}46.2 \text { (95\% Cl: } \\
26.6-66.6 \%)\end{array}$} & \multicolumn{2}{|c|}{$\begin{array}{c}53.8 \text { (95\% Cl: } \\
33.4-73.4 \%)\end{array}$} \\
\hline Specificity (\%) & \multicolumn{2}{|c|}{$\begin{array}{c}98.2(95 \% \mathrm{Cl}: \\
96.8-99.0 \%)\end{array}$} & \multicolumn{2}{|c|}{$\begin{array}{l}96.8 \text { (95\% Cl: } \\
95.1-98.0 \%)\end{array}$} \\
\hline \multicolumn{5}{|c|}{ Cross-sectional survey } \\
\hline Total & 10 & 2,041 & 10 & 2,041 \\
\hline $\mathrm{PCRPf}+$ & 6 & 4 & 6 & 4 \\
\hline PCR Pf- & 4 & 2,037 & 4 & 2,037 \\
\hline Sensitivity (\%) & \multicolumn{2}{|c|}{$\begin{array}{c}60.0 \text { (95\% Cl: } \\
26.2-87.8 \%)\end{array}$} & \multicolumn{2}{|c|}{$\begin{array}{c}60.0 \text { (95\% Cl: } \\
26.2-87.8 \%)\end{array}$} \\
\hline Specificity (\%) & \multicolumn{2}{|c|}{$\begin{array}{c}99.8(95 \% \mathrm{Cl}: \\
99.5-99.9 \%)\end{array}$} & \multicolumn{2}{|c|}{$\begin{array}{c}99.8 \text { (95\% Cl: } \\
99.5-99.9 \%)\end{array}$} \\
\hline
\end{tabular}

$\mathrm{Pf}=$ Plasmodium falciparum; $\mathrm{uRDT}=$ ultrasensitive rapid diagnostic test .

on paper-based forms by field workers, uploaded into an Excel (Microsoft Corporation, Redmond, WA) database, and then merged with the results of qPCR analysis using the unique participant identification number. The descriptive statistical analysis was carried out using Stata 15.1 (StataCorp, College Station, TX). Using qPCR as the reference standard, sensitivity and specificity were calculated. As the main target population for malaria control and elimination in this region is forest goers, a subgroup analysis was carried out on this group, defined as anyone who had reportedly slept overnight in the forest in the previous month.

Ethical approval for this study was obtained from the Cambodian National Ethical Committee for Human Research and the London School of Hygiene \& Tropical Medicine Ethics Committee. Participants were included in the study only if informed consent was obtained (parents or guardians provided consent for children younger than 18 years). Children older than 12 years were also required to provide oral assent for participation in addition to the consent of their parent or guardian.

In total, 2,729 individuals were tested using all three diagnostic tests: 678 during active case detection and 2,051 during the cross-sectional survey. The positivity rate for $P$. falciparum infections by qPCR analysis was $3.8 \%(26 / 678)$ and $0.5 \%(10 / 2,051)$ for active case detection and the cross-sectional survey, respectively. The CRDT and URDT results were compared with the qPCR results as the reference standard (Table 1). When used for active case detection, the sensitivity of the cRDT and URDT for $P$. falciparum malaria was $46.2 \%(95 \% \mathrm{Cl}$ : 26.6-66.6\%) and 53.8\% (95\% Cl: 33.4-73.4\%) and specificity was $98.2 \%$ (95\% Cl: $96.8-99.0 \%)$ and $96.8 \%$ (95\% Cl: 95.1-98.0\%), respectively $(P=0.0127)$. Ten samples were positive by qPCR and negative by both types of RDTs and 11 samples were negative by qPCR but positive by both types of RDTs. When used in the cross-sectional survey, the sensitivity for $P$. falciparum was $60.0 \%(95 \% \mathrm{Cl}: 26.2-87.8 \%)$ and specificity was 99.8\% (95\% Cl: 99.5-99.9\%) for both cRDT and uRDTs compared with the reference PCR $(P=1)$. Four samples tested positive by qPCR and negative by both RDTs and vice versa. Figure 1 shows the parasite densities by the RDT result. Sub-analysis conducted on just forest goers showed similar findings (results not shown).

To our knowledge, this is the first published study to report on the diagnostic performance of uRDTs under field conditions. The uRDTs were found to have similar sensitivity and specificity to both cRDTs when used in active case detection and in a cross-sectional survey. This differs from what has been reported from laboratory-based studies. Das et al. ${ }^{9}$ reported a uRDT sensitivity of $44 \%(95 \% \mathrm{Cl}$ : $15-77 \%)$ and specificity of $99.8 \%(95 \% \mathrm{Cl}: 99-100 \%)$ compared with quantitative reverse transcriptase PCR (qRT-PCR) and compared with a sensitivity of $0 \%(95 \% \mathrm{Cl}$ : $0-37 \%)$ and a specificity of $100 \%(95 \% \mathrm{Cl}$ : 99-100\%) for the CRDT (Bioline, Standard Diagnostics) in whole blood specimens collected from asymptomatic individuals in a

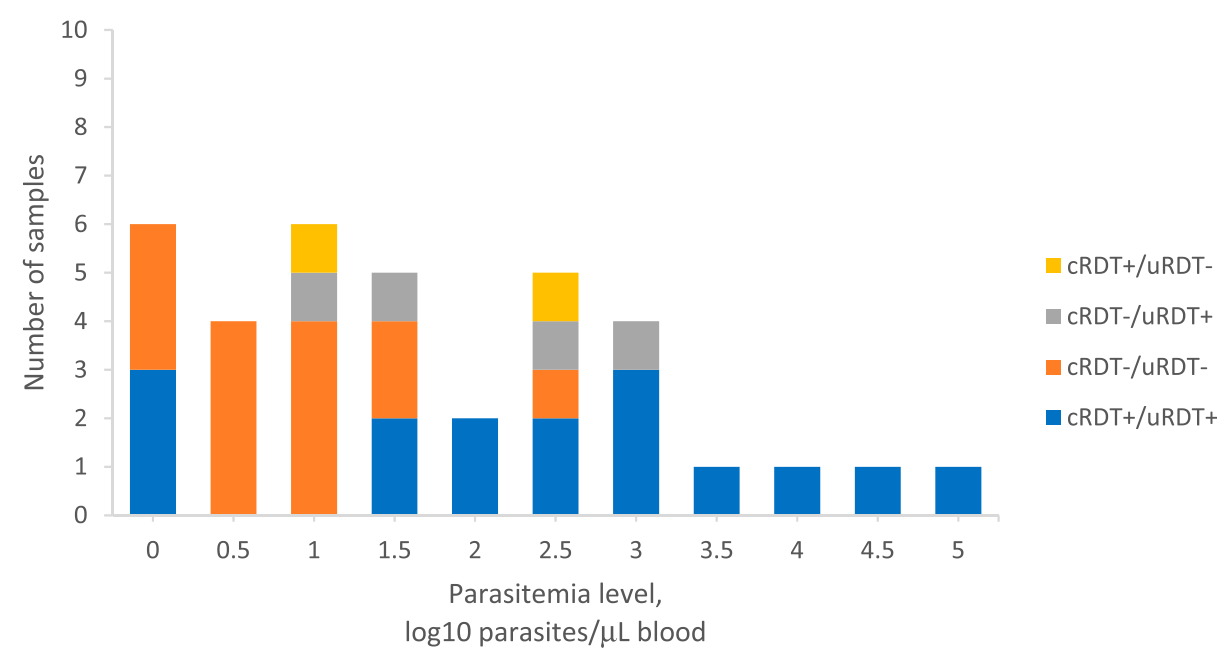

FIGURE 1. Distribution of estimated parasite density calculated from polymerase chain reaction cycle threshold levels, in comparison with conventional rapid diagnostic tests (RDTs) and ultrasensitive RDTs (uRDTs) for screening for Plasmodium falciparum during active case detection and cross-sectional survey in Cambodia. 
cross-sectional survey in Myanmar, where the positivity was $1.8 \%$ (9 of 493) by qRT-PCR. An analysis of 247 blood samples from a cross-sectional survey in Papua New Guinea by Hofmann et al. ${ }^{12}$ reported a uRDT (Malaria Ag P.f Ultra-Sensitive, Standard Diagnostics) sensitivity of $27 \%$ compared with $15 \%$ for cRDTs (Malaria Ag P.f/P.v, Standard Diagnostics) with qPCR used as a reference method. It should be noted that the PCR techniques used differed between studies and may explain some of the differences in the results.

There are a number of reasons why the uRDTs did not show enhanced sensitivity compared with cRDTs in this study. The uRDTs were stored in an air-conditioned office; however, it is possible that for brief periods during power cuts and transportation, they may have been exposed to temperatures of up to $35^{\circ} \mathrm{C}$-higher than the recommended maximum of $30^{\circ} \mathrm{C}$. Incorrect use by the field workers is also possible but very unlikely, as they received extensive training, were closely supervised and worked in pairs so that two people checked the results. A final consideration is the possibility of HRP2 deletions; however, this has not yet been reported in the region.

Despite their reported enhanced sensitivity, uRDTs did not show a significant improvement in diagnostic performance over cRDTs when used for active case detection and for a malaria prevalence survey in the context of this low-transmission setting. Further field evaluations for uRDTs are required in different epidemiological and under different operational settings to establish where they are likely to be of most benefit.

Received May 6, 2019. Accepted for publication October 1, 2019.

Published online December 9, 2019.

Acknowledgments: We thank warmly all the study participants, field teams, village malaria workers, and health center staff. We also thank Alere for donating uRDTs for the study.

Financial support: This work is funded by the United Kingdom Department for International Development (UKAID, agreement PO 5408).

Authors' addresses: Shunmay Yeung, David McGregor, and Nicola James, Clinical Research Department, Faculty of Infectious and Tropical Disease, Malaria Centre, London School of Hygiene and Tropical Medicine, London, United Kingdom, E-mails: shunmay.yeung@lshtm.ac.uk, mcgregor1@Ishtm.ac.uk, and nicola.h.james@gmail.com. Soy Ty Kheang, Health and Social Development (HSD), Phnom Penh, Cambodia, E-mail: ksoyty@urc-chs.com. Saorin Kim, Nimol Khim, and Benoit Witkowski, Institut Pasteur du Cambodge, Phnom Penh, Cambodia, E-mails: ksaorin@pasteur-kh.org, knimol@pasteur-kh.org, and bwitkowski@ pasteur-kh.org. Po Ly and Siv Sovannaroth, National Center for
Parasitology, Entomology and Malaria Control, Phnom Penh, Cambodia, E-mails: polyteng168@gmail.com and sivsovannaroths@ gmail.com.

This is an open-access article distributed under the terms of the Creative Commons Attribution (CC-BY) License, which permits unrestricted use, distribution, and reproduction in any medium, provided the original author and source are credited.

\section{REFERENCES}

1. Sochantha T, Hewitt S, Nguon C, Okell L, Alexander N, Yeung S, Vannara $H$, Rowland M, Socheat D, 2006. Insecticide-treated bednets for the prevention of Plasmodium falciparum malaria in Cambodia: a cluster-randomized trial. Trop Med Int Health 11: 1166-1177.

2. WHO, 2018. World Malaria Report 2018. Geneva, Switzerland: World Health Organization.

3. Ashley EA et al.; Tracking Resistance to Artemisinin Collaboration, 2014. Spread of artemisinin resistance in Plasmodium falciparum malaria. N Engl J Med 371: 411-423.

4. Ministry of Health Kingdom of Cambodia, 2017. Cambodia Malaria Elimination Action Framework (2016-2020) | MESA. Available at: http://mesamalaria.org/resource-hub/cambodiamalaria-elimination-action-framework-2016-2020. Accessed October 1, 2019.

5. Bousema T, Okell L, Felger I, Drakeley C, 2014. Asymptomatic malaria infections: detectability, transmissibility and public health relevance. Nat Rev Microbiol 12: 833-840.

6. Gryseels $C$ et al., 2015. High mobility and low use of malaria preventive measures among the Jarai male youth along the Cambodia-Vietnam border. Am J Trop Med Hyg 93: 810-818.

7. Guyant P, Canavati SE, Chea N, Ly P, Whittaker MA, Roca-Feltrer A, Yeung S, 2015. Malaria and the mobile and migrant population in Cambodia: a population movement framework to inform strategies for malaria control and elimination. Malar J 14: 252.

8. Bannister-Tyrrell $M$ et al., 2019. Forest goers and multidrugresistant malaria in Cambodia: an ethnographic study. $\mathrm{Am} \mathrm{J}$ Trop Med Hyg 100: 1170-1178.

9. Das $S$ et al., 2017. Performance of a high-sensitivity rapid diagnostic test for Plasmodium falciparum malaria in asymptomatic individuals from Uganda and Myanmar and naive human challenge infections. Am J Trop Med Hyg 97: 1540-1550.

10. Hoyer $S$ et al., 2012. Focused screening and treatment (FSAT): a PCR-based strategy to detect malaria parasite carriers and contain drug resistant $P$. falciparum, Pailin, Cambodia. PLoS One 7: e45797.

11. Canier L et al., 2015. Malaria PCR detection in Cambodian lowtransmission settings: dried blood spots versus venous blood samples. Am J Trop Med Hyg 92: 573-577.

12. Hofmann NE et al., 2018. Assessment of ultra-sensitive malaria diagnosis versus standard molecular diagnostics for malaria elimination: an in-depth molecular community cross-sectional study. Lancet Infect Dis 10: 1108-1116. 